\title{
Robust Automated Airbag Module Calibration
}

\author{
Peter J. Schubert \\ Delphi Delco Electronics Systems
}

Reprinted From: Airbag Technology 2001

(SP-1615) 
The appearance of this ISSN code at the bottom of this page indicates SAE's consent that copies of the paper may be made for personal or internal use of specific clients. This consent is given on the condition, however, that the copier pay a $\$ 7.00$ per article copy fee through the Copyright Clearance Center, Inc. Operations Center, 222 Rosewood Drive, Danvers, MA 01923 for copying beyond that permitted by Sections 107 or 108 of the U.S. Copyright Law. This consent does not extend to other kinds of copying such as copying for general distribution, for advertising or promotional purposes, for creating new collective works, or for resale.

SAE routinely stocks printed papers for a period of three years following date of publication. Direct your orders to SAE Customer Sales and Satisfaction Department.

Quantity reprint rates can be obtained from the Customer Sales and Satisfaction Department.

To request permission to reprint a technical paper or permission to use copyrighted SAE publications in other works, contact the SAE Publications Group.

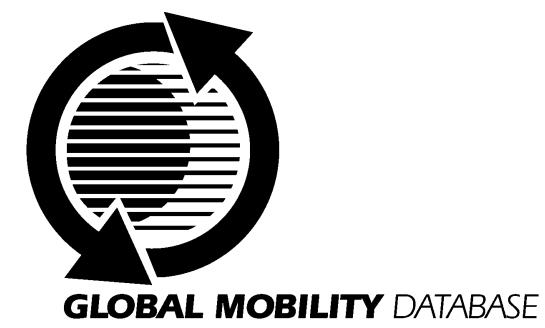

All SAE papers, standards, and selected books are abstracted and indexed in the Global Mobility Database

No part of this publication may be reproduced in any form, in an electronic retrieval system or otherwise, without the prior written permission of the publisher.

ISSN 0148-7191

Copyright 2001 Society of Automotive Engineers, Inc.

Positions and opinions advanced in this paper are those of the author(s) and not necessarily those of SAE. The author is solely responsible for the content of the paper. A process is available by which discussions will be printed with the paper if it is published in SAE Transactions. For permission to publish this paper in full or in part, contact the SAE Publications Group.

Persons wishing to submit papers to be considered for presentation or publication through SAE should send the manuscript or a 300 word abstract of a proposed manuscript to: Secretary, Engineering Meetings Board, SAE.

\section{Printed in USA}




\title{
Robust Automated Airbag Module Calibration
}

\author{
Peter J. Schubert \\ Delphi Delco Electronics Systems
}

Copyright (C) 2001 Society of Automotive Engineers, Inc.

\begin{abstract}
Increasing sophistication of electronic safety systems requires more advanced tools for design and optimization. Systems of safety products already being designed are becoming too interdependent to calibrate as stand-alone modules. Compounding this difficulty is the trend towards fewer test crashes and more sophisticated regulatory requirements. This paper presents a unified calibration approach to produce robust performance. First, the set of crash samples are extended using statistical techniques. Then an automated calibration tool using Genetic Algorithms is used to provide robust performance against deployment requirements. Finally, an expert systems is employed to ensure logical behavior. Together, these powerful methods yield calibrations which out-perform manual calibrations and can be completed in far less time.
\end{abstract}

\section{PROBLEM DESCRIPTION}

Automotive safety products must meet demanding requirements. Government regulations in the US market continue to move towards greater sophistication. Market forces are driving a trend towards safer vehicle operation through sensing devices, decision algorithms, restraint devices, and active safety measures [1]. With increasing numbers of such products on each vehicle, and with increasing interaction between them, the design, optimization, and validation of vehicle systems is becoming more challenging. As the requirements and difficulties mount, at the same time, there is intense pressure for systems to be provided at low cost, high reliability, and with short development cycle times. These opposing forces clearly call for a new paradigm in product development.

This study focuses on the development of crash sensing electronics. Crash testing, where expensive prototype or pilot vehicles are destroyed in the process, is an example of the tradeoffs involved in design needs versus budget cost. Design of a robust deployment algorithm, and calibration of that algorithm to a given vehicle platform requires a representative set of crashes.
Because of the wide variety of crashes that are possible, and the great variability even between repeated crashes, there is a strong need for a large library of crashes. To extract the most value from the limited set of crashes typically funded for a platform launch, several methods have been proposed [2], such as amplitude scaling, time scaling (with constant velocity), or noise addition. This paper presents a simple method of extending crash libraries to facilitate more robust calibrations for crash sensing algorithms.

The calibration process itself is accomplished through the careful setting of adjustable parameters within an algorithm to meet desired deployment decisions and timings, given the crash library. Calibration is a laborious process, and given the often-conflicting requirements, difficult to optimize. Delphi has developed an automated optimization routine using Artificial Intelligence and highperformance computing to greatly accelerate the calibration process, at the same time achieving higher quality results.

Validation of calibration results depends heavily on a peer review process, conducted by experts with considerable experience in this area. In this paper, we report on the use of a rules-based peer review tool system, which codifies the knowledge of these experts, thus streamlining the validation process and reducing the number of errors.

\section{METHODS}

CRASH LIBRARY EXTENSION - Ideally, a representative set of crash events would include low and high speed deploy tests, impacts into different barriers at different angles and varying amounts of offset to that barrier. Also important are non-deploy tests such as low speed impacts, rough roads and abuse events, representative of the many varied conditions that drivers force their vehicles to undergo. Such an ideal library would include hundreds of events, many of which destroy the vehicle. Clearly, this is impractical. 
The most common method to extend a crash library is to scale the amplitude of an accelerometer signal either higher or lower in magnitude to represent crashes of different speeds. This is a simple practice, but does not adequately reflect reality, since the dynamics of a crash event are highly non-linear. Another method is to scale the time axis of a crash, adjusting the amplitude so the crash energy remains constant. However, this practice is also a linear method, and is not supported by crash physics. Other methods use various bandpass filters to mix-and-match signals to poduce new test cases [2]. Noise addition is a further technique, where signalderived noise is added to a heavily-filtered signal [3]. These practice helps ensure algorithms are more robust against unavoidable crash variability.

For this work, we use a simple point-wise interpolation between crashes. Crash data from two target crashes is sampled at $10 \mathrm{kHz}$ and, at each data point, intermediate values are calculated. This method has the advantage that integrated velocity is bounded by the velocities of the two original crashes. Extrapolation techniques, such as amplitude scaling, time-scaling, and mix-and-match are not bounded, and can generate high-amplitude signals that may lead to undesired algorithm behavior. With point-wise interpolation, intermediate points can be scaled to provide desired equivalent velocities. Also, the noise of an interpolated crash will tend to be different from either target crash due to the non-correlation of the two crashes. This variety aids in the training of the calibration, making the results less susceptible to noise influences. Figure 1 shows a sample of point-wise interpolation.

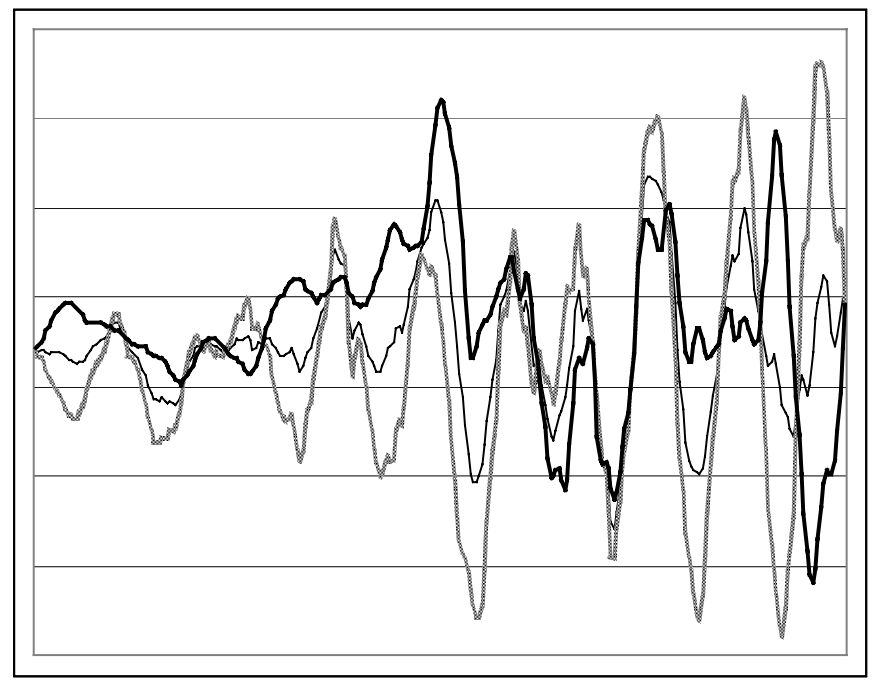

Figure 1. Crash signals for point-wise interpolation. Original (target) crashes are shown in thick black and thick gray lines, and the interpolated crashes are shown as a thin black line. Axes are purposely unlabeled for data anonymity.

The vehicle platform studied included only two no-trigger (NT) crash events, with 15 deploy tests. A crash set of this size leaves several regions of interest underrepresented. In particular, there was a gap of 10 kph between the fastest NT test and the slowest deploy test. To help fill in this region the low speed frontal and the frontal NT crashes were used to generate three intermediate points. For four types of deploy tests, crashes were performed at two different speeds, separated by either 13 or $18 \mathrm{kph}$, leaving significant gaps in the coverage of the crash universe.

\begin{tabular}{|l|l|l|l|l|}
\hline $\begin{array}{l}\text { Deploy } \\
\text { Tests }\end{array}$ & $\begin{array}{l}\text { Frontal } \\
\text { Hi speed }\end{array}$ & $\begin{array}{l}\text { Left } \\
\text { Angle } \\
\text { Hi Speed }\end{array}$ & $\begin{array}{l}\text { Right } \\
\text { Angle } \\
\text { Hi Speed }\end{array}$ & $\begin{array}{l}\text { Center } \\
\text { Pole } \\
\text { Hi Speed }\end{array}$ \\
\cline { 2 - 5 } & $\begin{array}{l}\text { Frontal } \\
\text { Lo speed }\end{array}$ & $\begin{array}{l}\text { Left } \\
\text { Angle } \\
\text { Lo Speed }\end{array}$ & $\begin{array}{l}\text { Right } \\
\text { Angle } \\
\text { Lo Speed }\end{array}$ & $\begin{array}{l}\text { Center } \\
\text { Pole } \\
\text { Lo Speed }\end{array}$ \\
\hline NT Test & $\begin{array}{l}\text { Frontal no } \\
\text { deploy }\end{array}$ & & & \\
\hline
\end{tabular}

Figure 2. Portion of crash set, showing sets of identical crash setups run at different speeds.

Point-wise interpolation was used for the Frontal, Left Angle, Right Angle and Center Pole events, shown in Figure 2, to generate intermediate crashes. Trigger times were defined by equivalent speed, and interpolated linearly between the high speed and the low speed deploy time requirements. In addition to these events, interpolation was also performed between the low speed center pole crash and an underride event of the same speed. A new NT event was generated in a similar manner between the frontal NT event and a similar speed NT test of a different type. A total of 10 new crashes were created using point-wise interpolation.

The sets of crashes were grouped into data sets for the purposes of calibration and evaluation. Figure 3 summarizes these groupings.

\begin{tabular}{|l|l|}
\hline Crash Set Name & \multicolumn{1}{|c|}{ Method Used } \\
\hline Original Data & $\begin{array}{l}\text { None. This is the test set used for } \\
\text { calibration, including 2 NT and 15 } \\
\text { deploy crashes. }\end{array}$ \\
\hline Evaluation Data & $\begin{array}{l}\text { None. This is the crash set used to } \\
\text { evaluate performance of the } \\
\text { calibrations. Results are reported on } \\
\text { combined Original and Evaluation crash } \\
\text { sets. }\end{array}$ \\
\hline Scaled Data & $\begin{array}{l}\text { Lo speed crashes scaled up to fill gaps } \\
\text { between like-crash types. Includes 5 } \\
\text { new crashes. }\end{array}$ \\
\hline Interpolated Data & $\begin{array}{l}\text { New crashes created by interpolating } \\
\text { between like-crash types, or between } \\
\text { same-speed crashes of different types. } \\
\text { Includes 10 new crashes. }\end{array}$ \\
\hline
\end{tabular}


Figure 3. Crash sets used in this study, and the method used to generate the crashes.

AUTOMATED CALIBRATION OPTIMIZATION - Manual calibration is a difficult task, involving analysis of crash signals, detailed understanding of the algorithm, and considerable trial and error adjustment of parameters to meet requirements. Automating this process has been successfully accomplished using the Artificial Intelligence technique known as Genetic Algorithms [4]. Genetic Algorithms (GA) search the space of parameter combinations to optimize the performance of the algorithm to the requirements. Two components essential to this process are a simulation of the algorithm, and a numerical measure of how well the requirements are met. A set of robustness functions were developed which analyze many aspects of algorithm performance, and return a robustness value. This robustness value, when optimized, indicates optimized results, and can be used as a figure of merit in assessing the quality of performance [5]. The exact nature of the robustness function is not germain to the general method presented here; it simply must provide numerical values which track monotonically with perceived performance.

We have implemented a GA optimization routine across a cluster of 23 workstations to achieve automated airbag deploy calibrations in as little as 4 hours [6]. A schematic of this SuperCluster is shown in Figure 4.

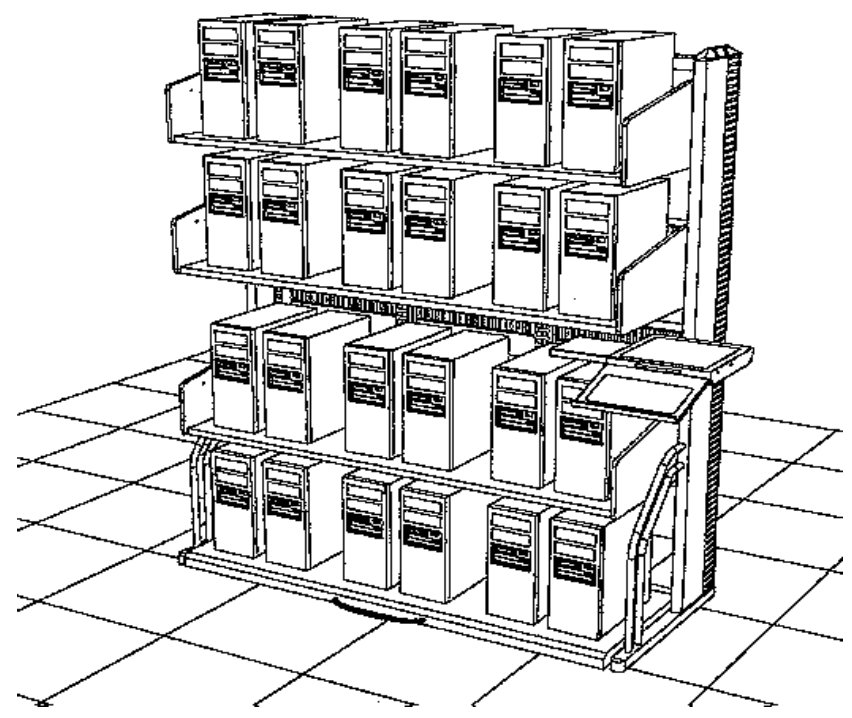

Figure 4. 23-Node SuperCluster of $450 \mathrm{MHz}$ Pentium II workstations using NT operating system, and a $100 \mathrm{Mb}$ Ethernet switch. Maximum operating speed is 10.6 gigaflops.

For the study reported here, we compare four different calibrations. The first method was a manual calibration, applied to the Scaled Data set, as described in Figure 3. The manual calibration employed a subset of the entire suite of functions available within the algorithm used.
This is considered a conservative approach, and is often used because simultaneous optimization of all 100-plus parameters is very challenging for a human calibration engineer.

The other three calibration methods each used the GA automated optimization tool, applied to, respectively: the Original Data set, the Scaled Data set, and the Interpolated Data set. The GA-optimized calibrations used all available parameters. From previous studies, we have found GA-optimized calibrations to perform equal or better than manual calibrations [6]. Each of the four calibrations was then applied to the Evaluation Data set without any further adjustment. The robustness value was computed, and the results compared to the robustness on the data set used for calibration.

PEER REVIEW EXPERT SYSTEM - Peer review of a calibration involves close analysis of results and the parameter settings used to achieve that output. With small crash libraries, a concern is that the calibration may perform well on the data available, but might give unwanted performance when exposed to crash signals beyond the training set. To guard against illogical or unwanted behavior, a peer review expert will pore over parameter settings, looking for values which, alone, or in combination with other parameters, may be inadvisable, based on their experience. To aid this process, and unburden the experts, a set of rules were coded in an expert system tool. The tool also allows the user to immediately run a robustness number check, providing instant feedback on the impact of adjusting parameters to the rule set. Occasionally we find that high performance parameter are outside of ranges the experts have investigated. In these cases, the rules file is updated, and distributed to the user community.

\section{RESULTS}

Figure 5 shows the results of this study by comparing the robustness value of the four methods of calibrations against their training data set, and against a common evaluation data set, which included 18 new crashes plus the original 17. Note that the robustness value ranges from 0.0 to 1.0 , with the latter being a perfect score. To account for tolerance of hardware and crash-to-crash variation, a $15 \%$ margin was added to all crashes when computing the robustness number. This margin was applied on the low side of deploy crashes and on the high side of NT crashes. 


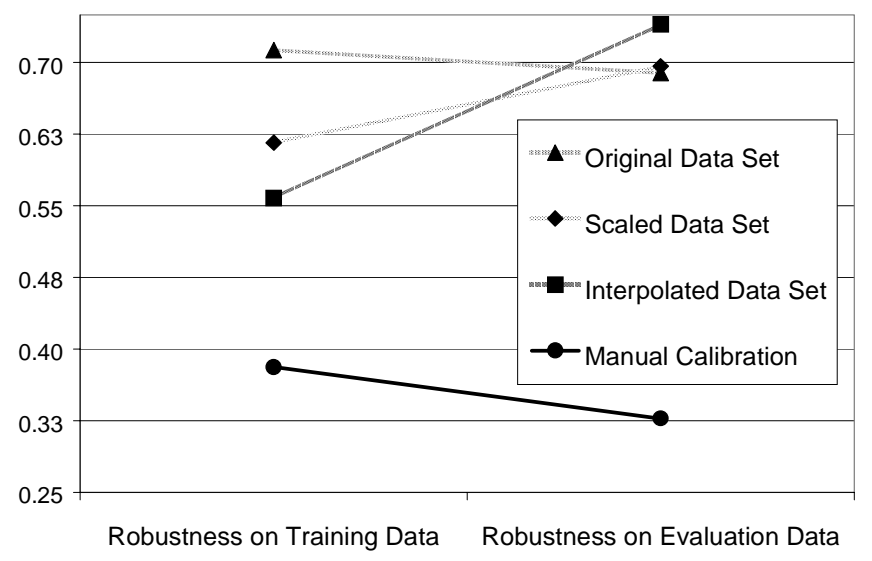

Figure 5. Robustness values for each calibration method, comparing results for the test data set and the evaluation data set (which included the original crash set).

Comparing each parameter value to a rule is facilitated by a simple tool which applies a rules file to the parameter values. Often, these rules are heuristic guidelines based on experience. Examples of parameters include velocity thresholds and filter cutoff frequencies; and examples of rules include minimum velocity thresholds depending on the filter characteristics. To this tool, we have added the ability to immediately re-compute the robustness value, providing rapid feedback to the calibration engineer on the effect of each adjustment.

In this study, the manual calibration had just one rule violation, but of a benign nature. The GA-optimized calibrations had more rules violations that required adjustment of parameters. In this type of automated search, those parameters which do not affect the robustness value result can sometimes be left with settings which seem inappropriate. The expert system tool checks these settings, and provides the user recommended adjustments. In the case of the GAoptimized calibration on the Interpolated Data set had three rules violations. When these were adjusted, the final robustness number did not change.

\section{DISCUSSION}

The true test of field-relevant robustness of an airbag algorithm calibration is the performance on new data beyond that which was used in the training phase. This robustness is achieved through three elements: an adequate training set, a thorough calibration, and careful setting of parameters. In this study, we have developed automated tools for each of these tasks. The results in Figure 5 illustrate the value of this new approach.

There are two key assessments of robustness in this context. The first is the absolute magnitude of the robustness value, which should be as close to 1.0 as possible. The second is the change in robustness value from the training set to the evaluation set. As can be seen in Figure 5, the GA-optimized calibrations for both the Scaled Data and Interpolated Data sets actually experience an increase in robustness when exposed to the new data in the Evaluation Data set (which also included the Original Data set). This is highly desirable, and was actually not anticipated in this case, and is not expected in general. However, Evaluation Data sets are sometimes less difficult than Training Data sets, so such a result is not impossible. Using only the Original Data for the training phase with the GA provided a calibration which diminished slightly in performance on the Evaluation Data set.

Taking the final robustness value on the Evaluate Data set, the GA-optimized calibration using the Interpolated Data set gave superior results to all other methods. Of great significance here is that the robustness value on the Interpolated Data set was low, compared to the results on the Scaled Data or Original Data sets. This implies that the Interpolated Data is more challenging for the algorithm. Yet, the dramatic increase in robustness value on the Evaluation Data set from the Interpolated Data also implies that the interpolated data provides rigorous test cases that ultimately result in a more robust calibration. This is the result we have been seeking.

\section{CONCLUSION}

Using the automated tools for extending the crash library, for the calibration process, and for peer review greatly accelerates the turnaround time. These tasks, which might take one to two weeks for a calibration engineer, can now be accomplished in two days. Using interpolation for crash library extension allows more robust calibrations with fewer expensive crash tests required. And with the power of Artificial Intelligence, complex algorithms can be optimized to realize their full potential. The use of expert systems reduces errors, and helps codify experiential knowledge, so that it can be further built upon. These tools have become indispensable aids to the design and optimization of advanced airbag modules, and provide a strong foundation for further advances in technology and requirements.

\section{ACKNOWLEDGMENTS}

The author extends grateful appreciation to Mark Feller of MGA for tools development, to Chris Caruso and Russell Simpson of Delphi for expert experience, to Kristi Reprogle of EDS for tools development, and to Dr. Dan Loughlin of the North Carolina State University for GA programming. 


\section{REFERENCES}

1. Bailey, R.S., and Runkle, D.L., "Delphi: A System of Systems," Automotive Engineering, May 2000, pp. 132-134.

2. Nusholtz, G.S., Shi, Y., and Xu, L., "Optimization of Single-Point Frontal Airbag Fire Threshold," SAE 2000 World Congress, Mar 6-9, 2000, 2000-01-1009.

3. Cuddihy, M.A., et. al, "Vehicle Crash Data Generator," US Patent 5,608,629, Mar. 4, 1997.

4. Goldberg, D.E., "Genetic Algorithms in Search, Optimization and Machine Learning:, Addison Wesley, 1989.

5. Schubert, P.J. and Loughlin, D.H. "Efficient Optimization of Large k Real-time Control Algorithm," in Proceedings of the Seventh International Workshop on Uncertainty in Statistics and Artificial Intelligence, Ft. Lauderdale, Jan 4-6, 1999.

6. Loughlin, D.H., and Schubert, P.J., "Calibrating Automotive Airbags Using a Genetic Algorithm", Interfaces, in publication.

\section{CONTACT}

Peter J. Schubert, Ph.D.

Restraint Systems Electronics

Delphi Delco Electronics Systems

Kokomo, Indiana 46904-9005

765-451-5925 fax 3600

peter.j.schubert@delphiauto.com 